Research Article

\title{
Performance Analysis and Optimization of a 6-DOF Robotic Crusher
}

\author{
Guoguang Li $\mathbb{D}^{1},{ }^{1}$ Boqiang Shi $\mathbb{D},{ }^{1}$ Ruiyue Liu $\mathbb{D}$, ${ }^{1}$ and Jie Gu $\mathbb{D}^{2}$ \\ ${ }^{1}$ School of Mechanical Engineering, University of Science and Technology Beijing, Beijing 100083, China \\ ${ }^{2}$ Beijing Institute of Space Launch Technology, Beijing 100076, China \\ Correspondence should be addressed to Boqiang Shi; shiboqiang@ustb.edu.cn
}

Received 5 August 2020; Accepted 19 October 2020; Published 4 November 2020

Academic Editor: Keigo Watanabe

Copyright ( 2020 Guoguang Li et al. This is an open access article distributed under the Creative Commons Attribution License, which permits unrestricted use, distribution, and reproduction in any medium, provided the original work is properly cited.

Considering the complexity of multidimension parameters and the mechanical performance of a 6-DOF robotic crusher, a multiobjective optimization function based on the transmission index and condition number is established. As an important operation in the screw theory, the reciprocal product between the transmission wrench screw of an actuator and the output twist screw of the mantle assembly is used to represent the instantaneous power. The expression of transmission index is derived according to the principle that constraint wrench screws apply no work to the mantle assembly. It can be used as a criterion to evaluate the transmission performance. Then, based on the Jacobian matrix, the equation of condition number is constructed which provides a criterion for evaluating kinematic accuracy. Finally, the workspace and singularity of the 6-DOF robotic crusher are analyzed to verify the rationality of the optimized variables. The results show that the optimized structure can completely crush the material in the workspace and effectively avoid singularity, which provides a basis for practical application.

\section{Introduction}

Crushers are widely used in the mining, mineral, road construction, and other industrial sectors to crush a variety of raw materials [1-3]. According to the different structural types, the commonly used crushers can be divided into the jaw crusher, gyratory crusher, and cone crusher [4]. Due to decades of theoretical research, they have evolved from simple crushers to modern high-performance crushers, which means that the structure becomes more complex and functions become more comprehensive [5]. Based on the interparticle breakage and population balance theory, the feed particles are squeezed and crushed not only by surfaces of the mantle and bowl liner but also by the surrounding particles. However, the design development of crushers still has some limitations under the present technology. They are inevitable to squeeze and crush materials with high power consumption and low flexibility due to the structural characteristics. Meanwhile, the parallel robot has attracted great attention due to its large payload, high-speed capability, and high stiffness [6-8]. It has been widely used in medical equipment, aerospace, marine, and other fields, which greatly promote the development of the industry. The intelligence of modern crushers must be a trend for future development.

Based on the above consideration, a novel 6-DOF robotic crusher was proposed which has achieved both the chamber structure of a cone crusher and the high flexibility of a parallel robot [9]. In Figure 1, the 6-DOF robotic crusher is composed of a drive unit (CDU) and a fixed unit (CFU). Figure 2 shows the mantle frame $\left\{O_{1}, X_{1}, Y_{1}, Z_{1}\right\}$ and the reference frame $\{O, X, Y, Z\}$. CDU mainly provides the power to crush the material, while $\mathrm{CFU}$ is fixed to the ground. The crushing process of the material in the crushing chamber can be represented by a number of crushing zones. The output of the previous crushing zone is the input to the next crushing zone. Then, the final product is excluded from the open side setting (OSS) due to gravity [10-12].

Undoubtedly, good structure design can greatly improve the performance of the 6-DOF robotic crusher. In order to evaluate the motion/force transmission of mechanisms, several important indices have been proposed, such as 


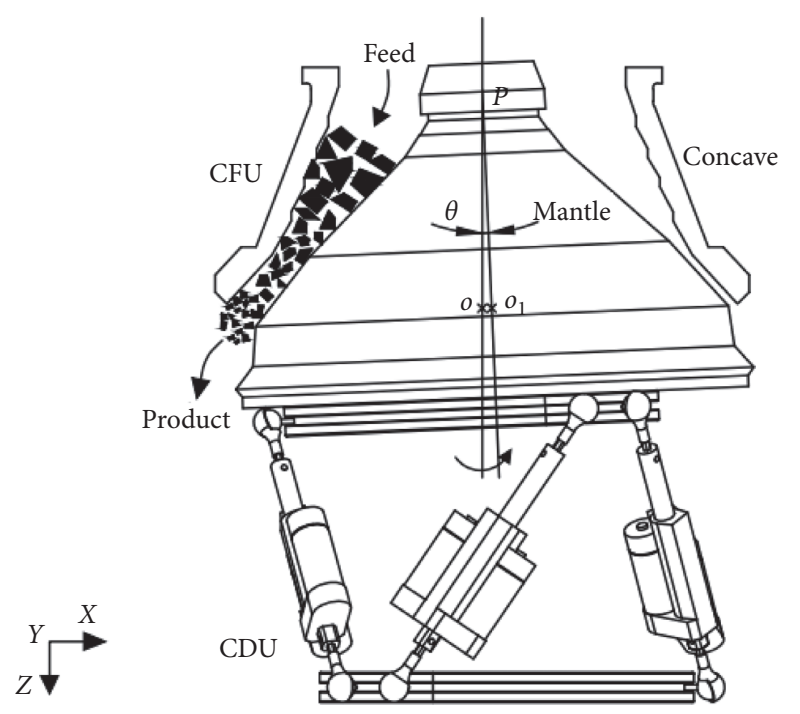

FIGURE 1: Schematic of the 6-DOF robotic crusher.

transmission angle, pressure angle, and transmission factor [13]. The transmission angle has been widely introduced and developed in the fields of planar mechanisms [14]. However, it cannot be used to define the 6-DOF robotic crusher due to the multiloop mechanism. The pressure angle is only suitable to evaluate the transmission of cam mechanisms, which makes its application receive little attention. The transmission index of the 6-DOF robotic crusher is used to quantitatively measure the effectiveness of the instantaneous power from the actuators to the mantle assembly. In order to achieve the required motion of the mantle assembly, the motion from the actuators needs to be transmitted to the mantle assembly. Meanwhile, the 6-DOF robotic crusher should transmit generalized forces from the mantle assembly to the actuators for the purpose of balancing the payload and gravity of the mantle assembly $[15,16]$. As the condition number of the Jacobian matrix represents the error amplification factor, it can be used as an index to measure the kinematic accuracy of the 6-DOF robotic crusher. The equation of the condition number is a function of the structural parameters, which can be changed with the variety of position and orientation in the workspace $[17,18]$. Based on the consideration of improved transmission performance and kinematic accuracy, a genetic algorithm is proposed to design the 6-DOF robotic crusher.

The workspace and singularity are very important issues and measurement criteria in the optimization design of the 6-DOF robotic crusher. The main difficulties are that the complete boundaries should be determined in a six-dimensional space. Due to the different fixed parameters, the workspace is divided into the position workspace and orientation workspace [19]. Considering the complexity of analytical expression, the polar coordinate method can be used to determine the trajectory boundary of the workspace directly. Meanwhile, the mathematical model for the singularity locus is obtained by using the force Jacobian matrix $[20,21]$. The singularity of the 6-DOF robotic crusher leads to the loss or gain of one or more degrees of freedom [22].

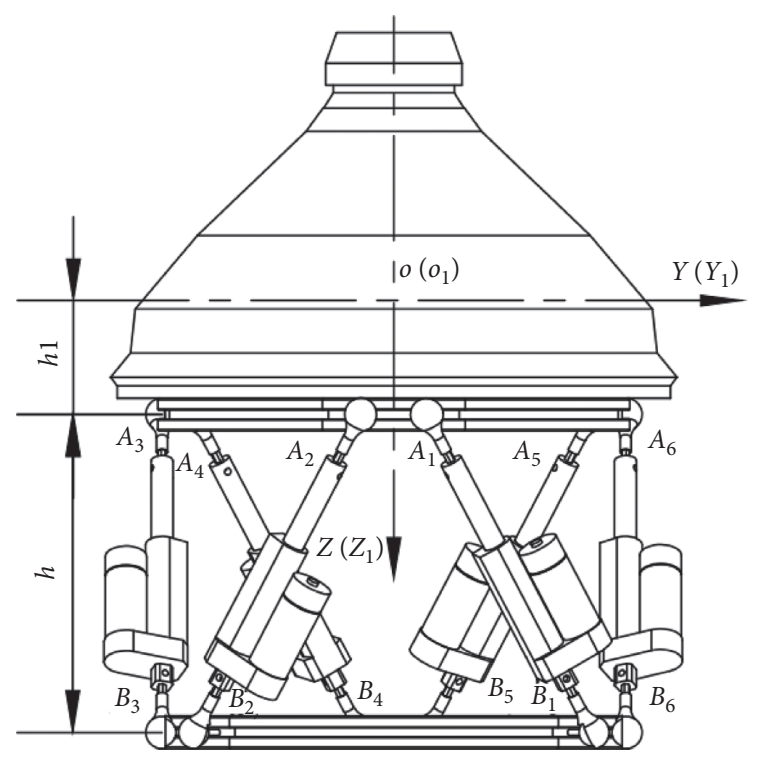

Figure 2: The model of CDU.

The graphical representation of the complete boundary can be displayed with a given set of fixed parameters. Then, it is easy to identify the boundary curves of the workspace and the singularity locus, so as to verify the rationality of optimization design.

\section{Transmission Performance}

The evaluation index can be used to quantitatively analyze the mechanical performance of the 6-DOF robotic crusher. Considering the importance of energy conservation and accuracy, transmission index and condition number are taken as research objects, which are both functions of structural parameters. Optimization design can undoubtedly improve production size distribution and increase productivity.

2.1. Screw Theory. Screw theory is an effective mathematical tool which can be used to analyze the mechanical performance of the 6-DOF robotic crusher. A screw can be shown by a set of a dual vector that represents the direction and position of an axis, respectively. According to mechanical characteristics, the screw may be divided into two categories: twist screw and wrench screw. The motion of the mantle assembly is described by the combination of a rotation around an axis and a movement along the axis, which can be represented as a twist screw. The twist screw of the mantle assembly can be expressed as

$$
\$_{t}=(\boldsymbol{\omega} ; \boldsymbol{v})=\left(\boldsymbol{\omega} ; \mathbf{r}_{1} \times \boldsymbol{\omega}+h_{\mathrm{t}} \boldsymbol{\omega}\right),
$$

where $\omega$ is the vector of angular velocity along the axis and $v$ is the linear velocity of the point that coincides with the origin on the mantle assembly. $\mathbf{r}_{1}$ represents the vector from the origin of the reference frame to any point on the axis, and $h_{\mathrm{t}}$ denotes the pitch of the twist screw. 
Similarly, the force and moment applied to the mantle assembly can be described by a wrench screw. The wrench screw can be shown as

$$
\mathbf{\$}_{w}=(\mathbf{f} ; \boldsymbol{\tau})=\left(\mathbf{f} ; \mathbf{r}_{2} \times \mathbf{f}+h_{\mathrm{w}} \mathbf{f}\right)
$$

where $\mathbf{f}$ represents the force vector along the axis and $\tau$ is the moment of the force system relatives to the origin. $\mathbf{r}_{2}$ denotes the vector from the origin of the reference frame to any point on the axis, and $h_{\mathrm{w}}$ is the pitch of the wrench screw.

The instantaneous power cannot be directly used to analyze and evaluate the transmissibility of the 6-DOF robotic crusher due to the difference in structure scale. The transmission forces of the actuators will become the constraint forces when the reciprocal product is equal to 0 . A transmission index is defined as the ratio between the actual instantaneous power and the maximum instantaneous power theoretically possible, which can be expressed as

$$
\eta=\frac{\left|\mathbf{\$}_{w} \circ \mathbf{\$}_{t}\right|}{\left|\mathbf{\$}_{w} \circ \mathbf{\$}_{t}\right|_{\max }}=\frac{\left|\left(h_{\mathrm{t}}+h_{\mathrm{w}}\right) \cos \theta_{i}-d_{i} \sin \theta_{i}\right|}{\sqrt{\left(h_{\mathrm{t}}+h_{\mathrm{w}}\right)^{2}+d_{i \max }^{2}}}
$$

where $d_{i}$ is the common perpendicular between the two screw axes, and $\theta_{i}$ denotes the angle of the two screw axes.

2.2. Transmission Index. The essence of the material breakage of the 6-DOF robotic crusher is the transmissibility of motion and force between the actuators and the mantle assembly. The pendulum movement of the mantle assembly in the crushing chamber is performed by the transmission wrench screw and output twist screw of the mantle assembly. The motion can be passed from the input to the output to complete the action required by crushing. Meanwhile, the crushing force and gravity of the mantle assembly can be balanced by the transmission wrench screws.

As shown in Figure 3, the axes of the transmission wrench screw and the input twist screw of each actuator are along the prismatic pair, and the axe of the output twist screw of the mantle assembly is floating in space. Assuming that the prismatic joint of $i$-th actuator is only driven, and all the others are fixed for the 6-DOF robotic crusher. Thus, the transmission wrench screw of the driven actuator becomes the only one that can make some contribution to the mantle assembly, while all other transmission wrench screws turn into five constraint wrenches. Then, the CDU of the 6-DOF robotic crusher can be regarded as a single-DOF parallel mechanism. Therefore, the expression between the constraint wrench screw and output twist screws can be shown as

$$
\$_{\mathrm{wi}} \circ \$_{\mathrm{tj}}=0, \quad(i, j=1,2,3, \ldots, 6, i \neq j) .
$$

According to the geometric analysis of the screw, the transmission wrench screw can be defined as

$$
\begin{aligned}
\mathbf{\$}_{\mathrm{wi}} & =\left(\mathbf{n}_{\mathbf{i}} ; \mathbf{O A}_{\mathbf{i}} \times \mathbf{n}_{\mathbf{i}}\right), \\
& =\left(m_{i, 1}, m_{i, 2}, m_{i, 3} ; m_{i, 4}, m_{i, 5}, m_{i, 6}\right), \quad(i=1,2,3, \ldots, 6) .
\end{aligned}
$$

where $\mathbf{n}_{\mathbf{i}}$ denotes the unit vector of the actuator, and $m_{i, 1}, \ldots, m_{i, 6}$ are Plücker coordinates of the axis of the wrench screw.

Similarly, the unit output twist screw of the mantle assembly can be represented as

$$
\begin{aligned}
\$_{\mathrm{tj}} & =\left(k_{j, 1}, k_{j, 2}, k_{j, 3} ; k_{j, 4}, k_{j, 5}, k_{j, 6}\right), \quad(j=1,2,3, \ldots, 6), \\
k_{j, 1}^{2}+k_{j, 2}^{2}+k_{j, 3}^{2} & =1,
\end{aligned}
$$

where $k_{j, 1}, \ldots, k_{j, 6}$ are Plücker coordinates of the axis of the twist screw.

Assuming that the prismatic pair of the first actuator is only driven, (4) may be rewritten as

$$
\$_{\mathrm{w} 1} \circ \mathbf{S}_{\mathrm{tj}}=0, \quad(j=2,3, \ldots, 6) .
$$

According to (3), (5), and (6), the absolute value of the reciprocal product for the first actuator is expressed as

$$
\begin{aligned}
\left|\$_{w 1} \circ \$_{t 1}\right|= & \left|\left(h_{1}+h_{2}\right) \cos \theta_{1}-d_{1} \sin \theta_{1}\right|, \\
= & \mid m_{1,4} k_{1,1}+m_{1,5} k_{1,2}+m_{1,6} k_{1,3}+m_{1,1} k_{1,4} \\
& +m_{1,2} k_{1,5}+m_{1,3} k_{1,6} \mid .
\end{aligned}
$$

Then, the reciprocal product of the remaining five actuators is obtained as

$$
\begin{gathered}
m_{1,4} k_{1,1}+m_{1,5} k_{1,2}+m_{1,6} k_{1,3}+m_{1,1} k_{1,4}+m_{1,2} k_{1,5} \\
+m_{1,3} k_{1,6}=0, \quad(i=2,3, \ldots, 6) .
\end{gathered}
$$

As the value of the pitch is independent of the origin selection, the pitch of the unit output twist screw $\$_{\mathrm{t} 1}$ can be defined as

$$
h_{\mathrm{t} 1}=k_{1,1} k_{1,4}+k_{1,2} k_{1,5}+k_{1,3} k_{1,6} .
$$

Since the transmission wrench screw of the first actuator is a pure force, the pitch of the wrench screw is given by

$$
h_{\mathrm{w} 1}=0 \text {. }
$$

Consequently, the axis of the twist screw $\$_{t 1}$ can be expressed as

$$
\mathbf{r} \times \mathbf{S}_{1}=\mathbf{S}_{1}^{0}-h_{\mathrm{t} 1} \mathbf{S}_{1}
$$

where $\mathbf{S}_{\mathbf{1}}=\left(k_{1,1}, k_{1,2}, k_{1,3}\right)$ and $\mathbf{S}_{\mathbf{1}}^{\mathbf{0}}=\left(k_{1,4}, k_{1,5}, k_{1,6}\right)$.

As the axes of two kinds of screws for each actuator are along the prismatic pair, the input transmission index is always equal to 1 which can be derived from the equation (3). Hence, the input transmission index and the output transmission index of the first actuator can be gained as

$$
\begin{aligned}
& \eta_{i 1}=1, \\
& \eta_{o 1}=\frac{\left|\left(h_{t 1}+h_{w 1}\right) \cos \theta_{1}-d_{1} \sin \theta_{1}\right|}{\sqrt{\left(h_{t}+h_{w}\right)^{2}+d_{1 \max }^{2}}},
\end{aligned}
$$

where $d_{1 \max }$ can be derived as follows: 


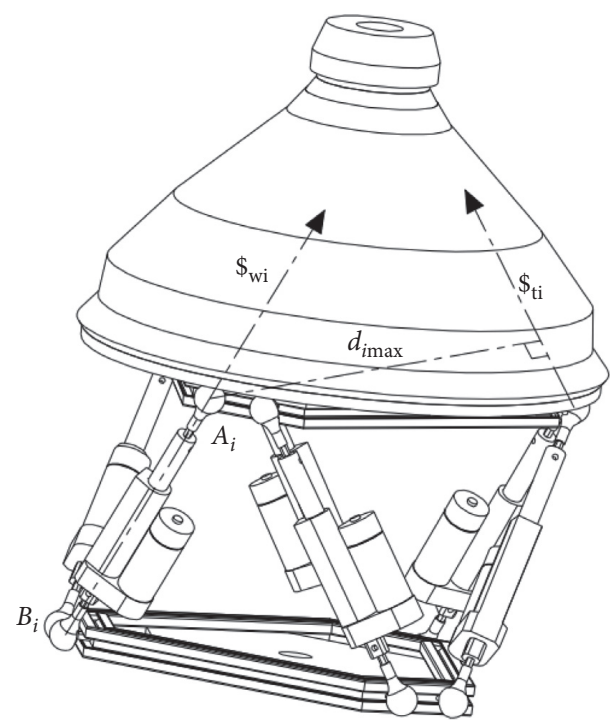

FIgURE 3: The position of $\$_{\mathrm{wi}}$ relative to $\$_{\mathrm{ti}}$.

$$
d_{1 \max }=\left|S_{1} \times L_{X}\right|
$$

where $L_{X}$ is the distance from $A_{1}$ to any point on the axis of output twist screw $\$_{\mathrm{t} 1}$, which can be obtained as

$$
L_{X}=|\mathbf{r}-\mathbf{O A}| \text {. }
$$

To sum up, a local transmission index can be used to evaluate the transmission performance of the 6-DOF robotic crusher, which is defined as

$$
\eta_{1}=\min \left\{\eta_{i 1}, \eta_{o 1}\right\}
$$

In order to unify the format of optimization design, (16) can be rewritten as

$$
f_{1}=-\eta_{1}
$$

2.3. Condition Number. The Jacobian matrix is the basis of kinematics and dynamics for the 6-DOF robotic crusher, which describes the relationship between the velocity of each actuator and the velocity of the mantle assembly. It is related to the structural parameters, which can be expressed as

$$
\dot{L}=\mathbf{J} \dot{P}=\mathbf{J}\left[\begin{array}{l}
v \\
\boldsymbol{\omega}
\end{array}\right],
$$

where $v$ is the translational velocity of the mantle assembly, $\omega$ represents the angular velocity of the mantle assembly, and $\mathrm{J}$ is the Jacobian matrix which can be expressed as

$$
\mathbf{J}=\left[\begin{array}{ll}
\mathbf{n}_{1} & \mathbf{r}_{\mathrm{a} 1} \times \mathbf{n}_{1} \\
\mathbf{n}_{2} & \mathbf{r}_{\mathrm{a} 2} \times \mathbf{n}_{2} \\
\mathbf{n}_{3} & \mathbf{r}_{\mathrm{a} 3} \times \mathbf{n}_{3} \\
\mathbf{n}_{4} & \mathbf{r}_{\mathrm{a} 4} \times \mathbf{n}_{4} \\
\mathbf{n}_{5} & \mathbf{r}_{\mathbf{a} 5} \times \mathbf{n}_{5} \\
\mathbf{n}_{6} & \mathbf{r}_{\mathrm{a} 6} \times \mathbf{n}_{6}
\end{array}\right],
$$

where $\mathrm{n}_{\mathrm{i}}$ is the unit vector of the $i$-th actuator, and $\mathrm{r}_{\mathrm{ai}}=\mathrm{TA}_{\mathrm{i}}$, $T$ is the transformation matrix of the mantle frame relative to the reference frame. $\mathrm{A}_{\mathrm{i}}$ represents the coordinate of $A_{i}$ in the mantle frame.

The actuator velocity will inevitably produce errors due to the accuracy issues of manufacturing and assembly, which leads to a corresponding error for the mantle assembly. The expression can be written as

$$
\dot{L}+\delta \dot{L}=\mathbf{J}(\dot{P}+\delta \dot{P}) .
$$

By subtracting (18) from (20), the relative error relation of the velocities between the actuators and the mantle assembly can be simplified as

$$
\frac{\|\delta \dot{P}\|}{\|\dot{P}\|} \leq k \frac{\|\delta \dot{L}\|}{\|\dot{L}\|}
$$

where $k$ represents the condition number of the Jacobian matrix, which is defined as

$$
k=\|\mathbf{J}\| \cdot\left\|\mathbf{J}^{-1}\right\| .
$$

The condition number of the Jacobian matrix is the amplification factor of the relative error, which can be used to evaluate the kinematic accuracy. The smaller the condition number, the higher the kinematic accuracy. Then, the following function within the unit period can be defined as

$$
f_{2}=-\frac{1}{k}
$$

where $1 \leq k \leq \infty$.

\section{Optimization and Performance Analysis}

3.1. Multiobjective Optimization. The genetic algorithm (GA) is a random search algorithm based on the biological evolution mechanism of natural selection and natural inheritance. Each value of the solution space should be coded first when solving optimization problems. New solutions are generated continuously by combining chromosomes. Thus, according to the fitness function, some chromosomes are selected in the new solutions to continue the combination until the best solution is finally found [23-26].

Here, the transmission index and condition number are taken as the optimization targets. The purpose of optimization design for the 6-DOF robotic crusher is to obtain good transmission performance and high kinematic accuracy, which can be regarded as a multiobjective optimization problem. Due to the different orders of magnitude between the two optimization targets, the multiobjective problem can be transformed into a single-objective problem. Then, the objective function can be shown as

$$
F=\frac{1}{l} \sum_{i=1}^{l}\left[\omega_{1}\left(\frac{f_{1}-f_{1}^{0}}{f_{1}^{0}}\right)^{2}+\omega_{2}\left(\frac{f_{2}-f_{2}^{0}}{f_{2}^{0}}\right)^{2}\right],
$$

where $\omega_{1}=0.7, \omega_{2}=0.3, f_{1}^{0}$ is the optimal value of the transmission index $f_{1}, f_{2}^{0}$ is the optimal value of the average of the condition number $f_{2}$, and $l$ is the number of sampling points. 
The transmission index and condition number can be determined when structural parameters are given. The structural parameters of the 6-DOF robotic crusher mainly include the radius $R_{a}$ of the circle formed by the upper hinge points, the smaller central angle $\delta_{a}$ of adjacent hinge points on the mantle assembly, the radius $R_{b}$ of the circle formed by the lower hinge points, the smaller central angle $\delta_{b}$ of adjacent hinge points on the base, and the height $h$ between the upper and lower centers. Thus, the design variables are defined as

$$
\mathbf{x}=\left[\begin{array}{lllll}
R_{a} & R_{b} & \delta_{a} & \delta_{b} & h
\end{array}\right]^{T},
$$

The distance between the center of mass of the mantle assembly and the circle formed by the upper hinge points is $104 \mathrm{~mm}$. Taking into account factors such as assembly and compactness of the 6-DOF robotic crusher, the constraint conditions of design variables should satisfy the following criteria:

$$
\left\{\begin{array}{l}
100<R_{a}<300 ; 200<R_{b}<800 \\
15^{\mathrm{o}}<\delta_{a}<60^{\circ} ; 15^{\mathrm{o}}<\delta_{b}<60^{\circ} \\
180<h<300
\end{array}\right.
$$

3.2. Workspace Analysis. The workspace is an important indicator to evaluate the activity space of the 6-DOF robotic crusher, which can be measured by the shape and volume. According to the difference of fixed parameters, the workspace is divided into position workspace and orientation workspace. The position workspace refers to the set of all possible positions by fixing the orientation angle, and orientation workspace is defined as the set of all attainable orientations of the 6-DOF robotic crusher about a fixed point.

Inverse kinematics is not only the basis of dynamics and control but also plays a crucial role in the determination of the workspace boundary. When the position and orientation of the mantle assembly are given, the actuator length can be shown as

$$
L_{i}=\sqrt{\left[\mathbf{p}+\mathbf{T} \mathbf{A}_{i}-\mathbf{B}_{i}\right]^{T}\left[\mathbf{p}+\mathbf{T} \mathbf{A}_{i}-\mathbf{B}_{i}\right]}, \quad(i=1, \ldots, 6),
$$

where $\mathrm{p}$ represents the position vector of the mantle frame regard to the reference frame, $A_{i}$ and $B_{i}$ are used to denote the coordinate of $A_{i}$ in the mantle frame and the coordinate of $B_{i}$ in the reference frame, respectively. $T$ is the rotation matrix between the mantle frame and reference frame, which can be expressed as

$$
T=\left[\begin{array}{ccc}
c \beta c \gamma, & \mathrm{s} \alpha \mathrm{s} \beta \mathrm{c} \gamma-\mathrm{c} \alpha \mathrm{s} \gamma, & \mathrm{s} \alpha \mathrm{s} \gamma+\mathrm{c} \alpha \mathrm{s} \beta \mathrm{c} \gamma, \\
\mathrm{c} \beta \mathrm{s} \gamma, & \mathrm{c} \alpha \mathrm{c} \gamma+\mathrm{s} \alpha \mathrm{s} \beta \mathrm{s} \gamma, & -\mathrm{s} \alpha \mathrm{c} \gamma+\mathrm{c} \alpha \mathrm{s} \beta \mathrm{s} \gamma, \\
-\mathrm{s} \beta, & \mathrm{s} \alpha \mathrm{c} \beta, & \mathrm{c} \alpha \mathrm{c} \beta,
\end{array}\right]
$$

where $\alpha, \beta$, and $\gamma$ are orientation parameters of the generalized coordinate, $s=\sin$, and $c=\cos$.

The solution methods of the workspace mainly include the analytical method and the numerical method. The analytical method is generally complicated and can be used to solve the workspace of parallel robots with few degrees of freedom. Since the constraint equations of the 6-DOF robotic crusher are nonlinear and complex, a numerical method should be considered to solve the problem. According to the geometrical description of the spatial mechanism, the geometric constraints that limit the workspace include actuator length, range of the spherical balls, and actuator interference [27, 28]. But the actuator length is the most important factor due to the small space range of the 6-DOF robotic crusher.

Based on the above constraints of the 6-DOF robotic crusher, the polar coordinate method is adopted to conduct the possible boundaries of the workspace by increasing the polar radius, polar angle, and axial distance. In the process of searching the orientation workspace with the polar coordinate method, it is difficult to search the orientation workspace because of the periodicity of trigonometric functions contained in the transformation matrix and the difficulty in determining the upper and lower boundaries of the orientation angle. Considering that polynomials are easy to conduct numerical calculation, the polynomial is used instead of a trigonometric function to calculate the boundaries, and the expression can be shown as

$$
\begin{aligned}
& \sin \alpha=\frac{2 p_{1}}{\left(1+p_{1}^{2}\right)}, \\
& \cos \alpha=\frac{\left(1-p_{1}^{2}\right)}{\left(1+p_{1}^{2}\right)}, \\
& \sin \beta=\frac{2 p_{2}}{\left(1+p_{2}^{2}\right)}, \\
& \cos \beta=\frac{\left(1-p_{2}^{2}\right)}{\left(1+p_{2}^{2}\right)}, \\
& \sin \gamma=\frac{2 p_{3}}{\left(1+p_{3}^{2}\right)}, \\
& \cos \gamma=\frac{\left(1-p_{3}^{2}\right)}{\left(1+p_{3}^{2}\right)},
\end{aligned}
$$

where $p 1=\tan (\alpha / 2), p 2=\tan (\beta / 2)$, and $p 3=\tan (\gamma / 2)$.

The flow chart of searching the boundary trajectory of the workspace can be shown in Figure 4.

3.3. Singularity Analysis. Singularity is one of the main problems in the analysis and design of the 6-DOF robotic crusher, which can block the transmission of the motion and force between the mantle assembly and actuators. The local degree of freedom becomes uncontrollable when the singular configuration is formed, and the specific form mainly includes two cases. One is that the 6-DOF robotic crusher gains additional degrees of freedom which results in a great 


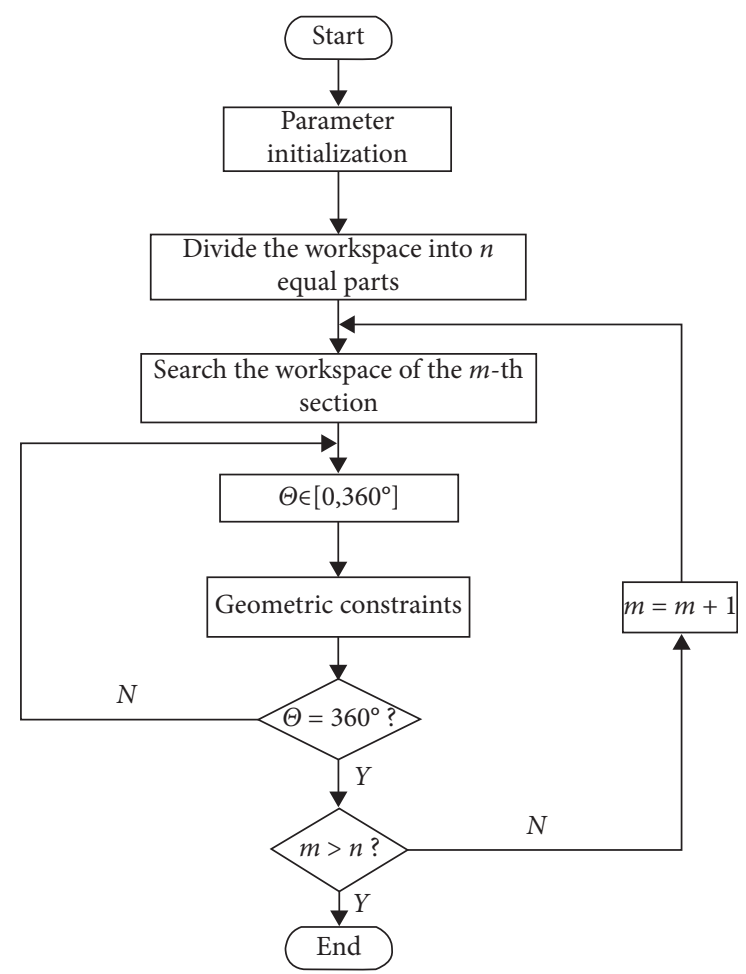

FIGURE 4: Flow chart of the workspace.

reduction in rigidity and carrying capacity. The other is that the 6-DOF robotic crusher loses several degrees of freedom, which leads to the loss of freedom in the uncontrollable direction. Therefore, the singular configuration of the 6DOF robotic crusher should be avoided in the crushing process of materials.

According to different fixed parameters, singularity can be divided into the position singularity for a constant orientation and orientation singularity at a constant position. It is necessary to get the analytic expression of the singularity locus from the point of view of design. Then, the graphical representation is obtained by a given set of structure parameters. Thus, the locations of singularity within the workspace can be easily identified. Due to the fact that the force Jacobian matrix of the 6-DOF robotic crusher describes the transformation relationship between the generalized forces of the mantle assembly and driving forces of the actuators, it can be used to determine the position singularity and orientation singularity. According to the geometric analysis, the expression of the force Jacobian matrix can be shown as $[29,30]$

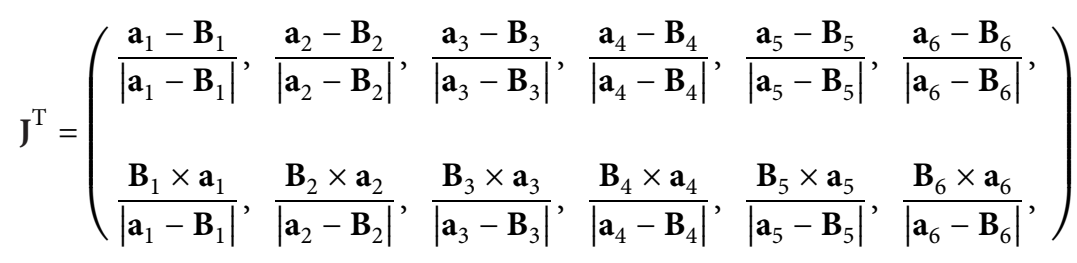

where $\mathrm{a}_{\mathrm{i}}$ and $\mathrm{B}_{\mathrm{i}}$ are the coordinates of $A_{i}$ and $B_{i}$ in the reference frame.

The equation of singularity locus for general orientations of the 6-DOF robotic crusher is derived by setting the determinant of the force Jacobin matrix to zero, which can be obtained as

$$
\begin{aligned}
C_{1} & Z^{3}+C_{2} X Z^{2}+C_{3} Y Z^{2}+C_{4} X^{2} Z+C_{5} Y^{2} Z \\
& +C_{6} X Y Z+C_{7} Z^{2}+C_{8} X Z \\
& +C_{9} Y Z+C_{10} X^{2}+C_{11} X Y+C_{12} Y^{2}+C_{13} Z \\
& +C_{14} Y+C_{15} X+C_{16}=0 .
\end{aligned}
$$


where $X, Y$, and $Z$ are position parameters of the generalized coordinate, equation (31) is a cubic polynomial function, and $C_{i}$ denotes a function of $\alpha, \beta$, and $\gamma$.

Extending and merging the determinant of the force Jacobian matrix when $Z$ is constant (31) can be rewritten as

$$
f(X, Y)=0 .
$$

Similarly, the singularity locus with different positions can be derived. The polynomial equation with respect to trigonometric functions of Euler angles is represented as

$$
f(\alpha, \beta, \gamma)=0
$$

Due to the periodicity of the trigonometric function, the orientation singularity is difficult to solve. However, polynomials are of great significance in the study of orientation singularity for the 6 -DOF robotic crusher because they can be used for numerical solutions. Thus, the orientation singularity can be deduced by substituting (29) into (33).

\section{Results and Discussion}

In this section, the structure parameters of the 6-DOF robotic crusher are optimized by the proposed GA methodology. Meanwhile, in order to evaluate the mechanical performance, the transmission index and condition number are calculated. Based on the analysis of the workspace and singularity, several sampling points were taken as examples to verify the validation of the optimization design.

4.1. Numerical Results. In Figure 5, the operation principle of the cone crusher is that the rotation of the eccentric drives the mantle to realize precession, and then particles are squeezed and crushed in the crushing chamber. The trajectory model of the mantle is the basic condition to determine the actual activity space and singularity of the 6DOF robotic crusher. Due to the high coupling degree of translation and rotation, the trajectory model should be considered to be established by Adams simulation. According to previous research, the trajectory model of the mantle frame with respect to the reference frame is represented as [9]

$$
\begin{aligned}
& \mathbf{q}_{\mathbf{e}}=\left[\begin{array}{c}
0.044 \sin (\omega t) \\
0.044 \cos (\omega t) \\
-0.078 \sin (\omega t)
\end{array}\right] \mathrm{rad}, \\
& \mathbf{q}_{\mathbf{p}}=\left[\begin{array}{c}
14.36 \cos (\omega t)-4.864 \\
-14.36 \sin (\omega t) \\
0
\end{array}\right] \mathrm{mm},
\end{aligned}
$$

where $\mathbf{q}_{\mathbf{e}}$ is the orientation trajectory model of the generalized coordinate, $\mathbf{q}_{\mathbf{p}}$ is the position trajectory model of the generalized coordinate, and $\omega=1.483 \mathrm{rad} / \mathrm{s}$.
According to the GA methodology, the optimized value of the structure parameters for the 6-DOF robotic crusher is shown in Table 1 . It can be found that the radius $R_{b}$ and angle $\delta_{b}$ of the lower hinge points are both greater than $R_{a}$ and $\delta_{a}$ of the upper points. Figure 6 shows the relationship between the fitness value and genetic algebra. The fitness function presents a downward trend during the whole iteration, and it converges rapidly in the initial stage of optimization. Then, the convergence rate slows down until the best fitness is reached, at which point the fitness function tends to the horizontal line. The entire optimization process has experienced 220 generations of genetic iterations. The result demonstrates that the GA methodology for the 6-DOF robotic crusher has the ability of fast optimization.

Since the trajectory model of the mantle along the $Z$-axis is given as $Z=0$, the distribution of the transmission index and condition number can be shown in Figure 7 when fixing the orientation angle $(\alpha, \beta, \gamma)=\left(2.52^{\circ}, 0^{\circ}, 0^{\circ}\right)$. The larger the transmission index, the better the transmission performance, and it is easy to find that the optimal location is around $[X, Y]=[-170,0]$. The condition number is used to evaluate the kinematic accuracy. The smaller the condition number, the higher the kinematic accuracy. In the Atlas, the optimal location is near $[X, Y]=[0,0]$. The common point of the two indexes is that the mechanical performance becomes worse when they are far away from the centers of the contour line.

According to the optimized structural parameters and combined with equations (16) and (34), the variation law of the transmission index of the 6-DOF robotic crusher with time can be obtained through numerical simulation. The variation rule of the conditional number can be gained by numerical simulation of equations (22) and (34). As shown in Figure 8, the transmission index changes periodically in the form of a trigonometric function, and the worst value is greater than 0.7 . It indicates that the 6-DOF robotic crusher has good motion/force transmission performance and is far away from its singularity. Meanwhile, the variety curve of the condition number during the whole period appears in an $M$ shape. The value of kinematic accuracy is the highest at the beginning and the end. Due to the different time of maximum values, the weighting factors of two optimization targets can be adjusted to satisfy the working requirements of the 6-DOF robotic crusher.

4.2. Verification. In order to verify the rationality of the optimization design described above, the workspace and singularity of the 6-DOF robotic crusher are studied. Based on the boundary of the trajectory model of equation (34), it can be known that the intervals of the generalized coordinates are given as follows: $X \in(-20,10), Y \in(-15,15)$, $\alpha \in\left(-3^{\circ}, 3^{\circ}\right), \beta \in\left(-3^{\circ}, 3^{\circ}\right), \gamma \in\left(-5^{\circ}, 5^{\circ}\right)$. Here, the shortest length of the actuator is $283 \mathrm{~mm}$ and the stroke is $150 \mathrm{~mm}$.

In the process of the polar coordinate search for the position workspace of a 6 -DOF robotic crusher, the possible values of $Z$ direction are divided into $n$ equal parts with a plane parallel to the $X Y$ plane. According to the geometric constraints, starting from the minimum 


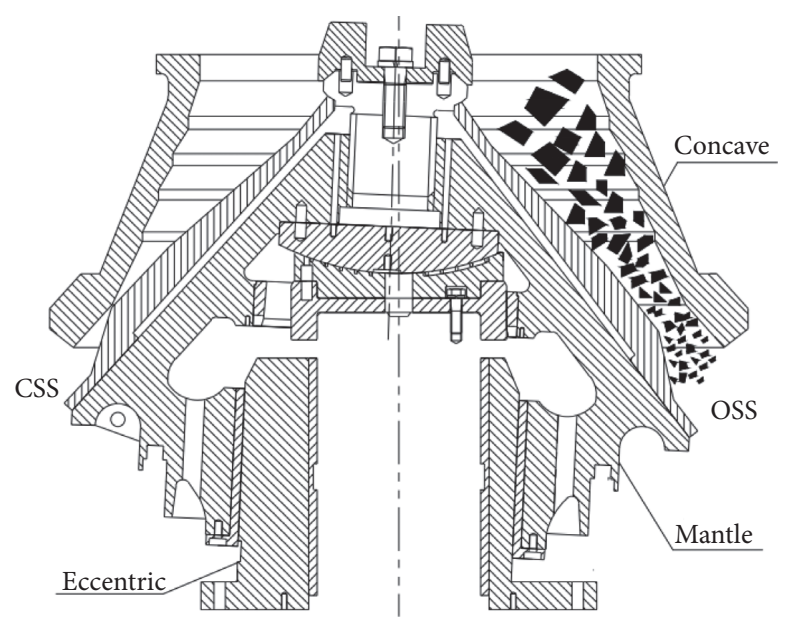

Figure 5: Cross section of cone crusher.

TABLE 1: The structure parameters optimized by GA.

\begin{tabular}{lr}
\hline Parameter & Value \\
\hline$R_{a}(\mathrm{~mm})$ & 100 \\
$R_{b}(\mathrm{~mm})$ & 299 \\
$\delta_{a}(\mathrm{rad})$ & 0.263 \\
$\delta_{b}(\mathrm{rad})$ & 0.783 \\
$h(\mathrm{~mm})$ & 225 \\
\hline
\end{tabular}

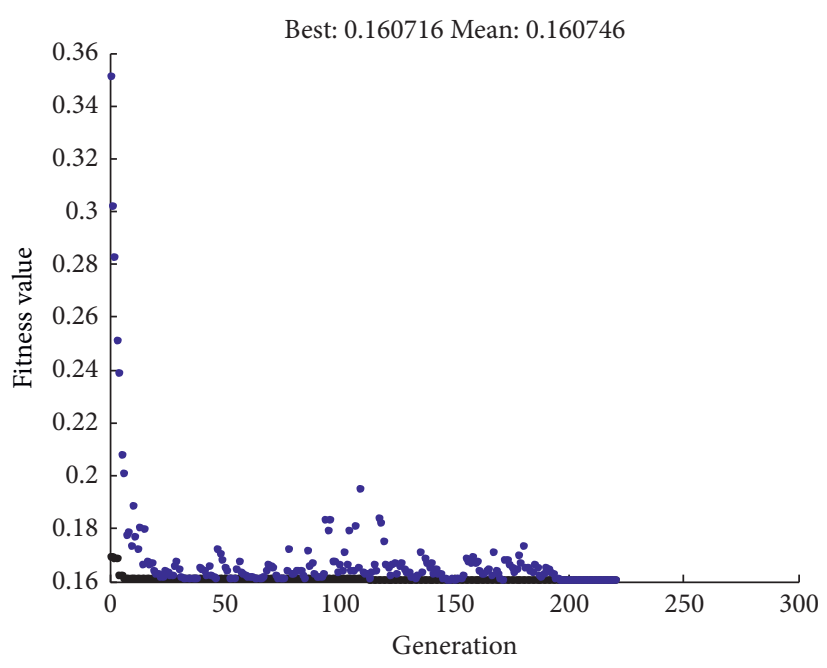

- Best fitness

- Mean fitness

Figure 6: Evolutionary process of fitness value.

value of the $Z$-axis and using the constant update of the polar angle and polar diameter, the search for the trajectory boundary of each subspace with a given orientation is realized. After the subspace is searched, we select the increment $\Delta Z$ along the $Z$ direction to continue searching until the maximum value of $Z$ is reached. As shown in Figure 9, the boundary of position workspace is obtained according to the algorithms in Section 3.2. Since the height difference between the mantle frame and reference frame is increased by $45 \mathrm{~mm}$ after optimization, the boundary of the $X Y$ plane can be described by the cross section of $Z=-45 \mathrm{~mm}$. Average sampling is carried out for the unit period of the trajectory model to obtain the position workspace of each orientation. It can be seen that the intervals of the generalized coordinates are all included in the boundaries of the position workspace. 


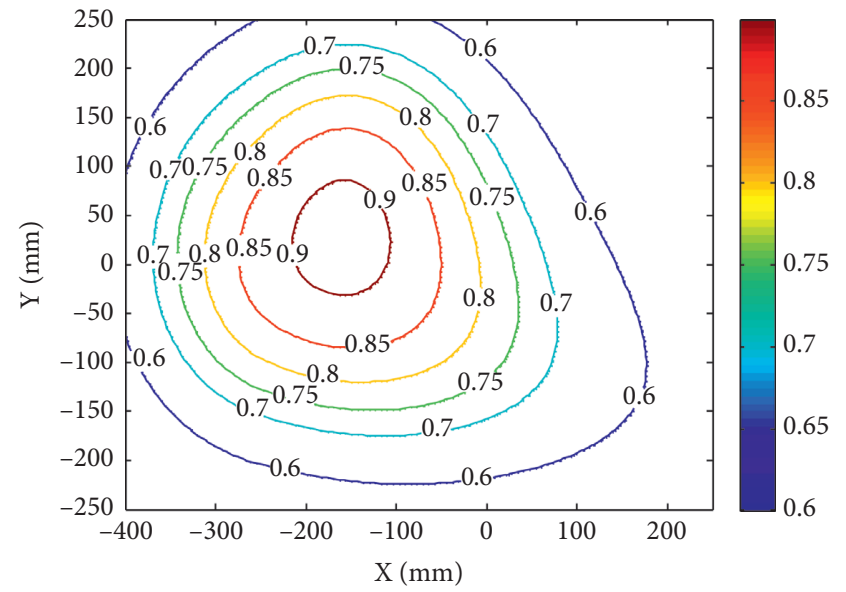

(a)

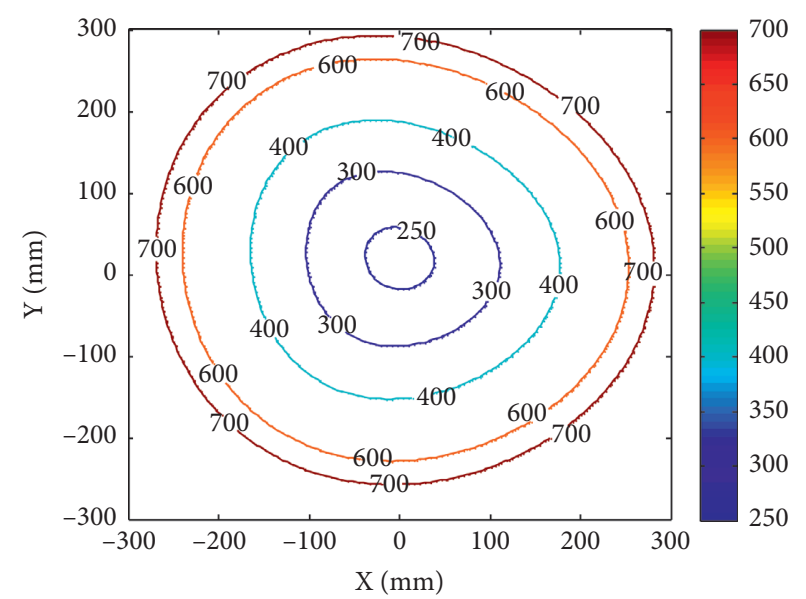

(b)

Figure 7: Distribution of the multiobjective functions by fixing the orientation angle $\left(2.52^{\circ}, 0^{\circ}, 0^{\circ}\right)$. (a) Transmission index. (b) Condition number.

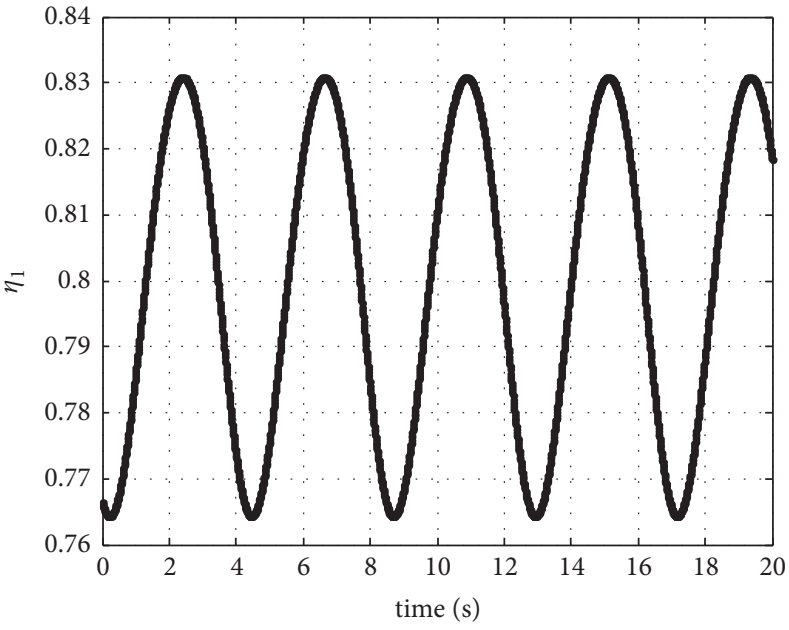

(a)

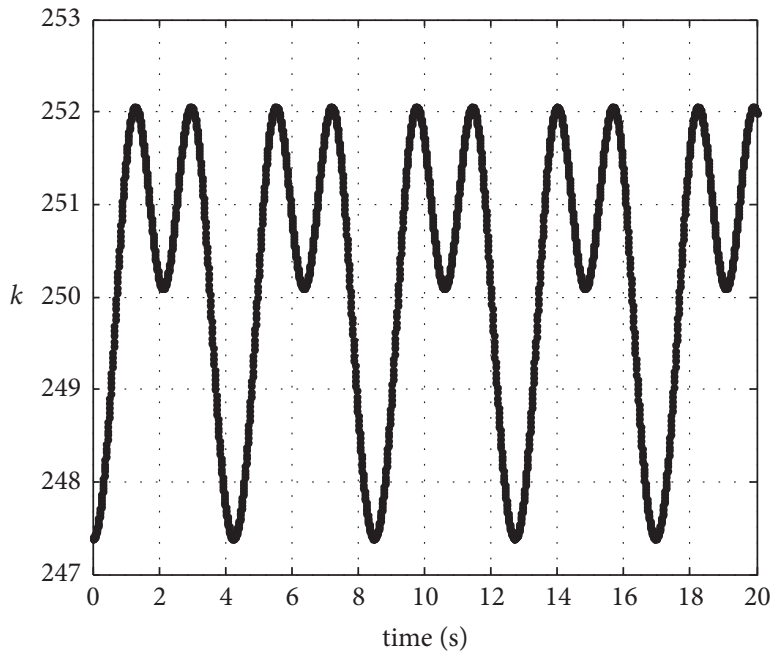

(b)

Figure 8: Multiobjective functions with time. (a) Transmission index. (b) Condition number.

The boundary curves of the orientation workspace are represented by fixing the position. In the process of searching the orientation workspace with the polar coordinate method, the polynomial in equation (29) is used to replace the trigonometric function for any given position $(X, Y, Z)$. The possible values of $p_{3}$ are divided into $n$ equal parts, and the search starts from the minimum value. The trajectory boundary search of each subspace is realized by using the continuous updating of the polar angle and polar diameter. We convert the $\left(p_{1}, p_{2}, p_{3}\right)$ that satisfy the orientation workspace boundary into the corresponding $(\alpha, \beta, \gamma)$. After each subspace completes the search, we take the increment in the $p_{3}$ direction to continue the search until it reaches the maximum value. According to the trajectory model, parameter $Z=0$ is fixed, and other parameters are averagely sampled in the unit period. The orientation workspace of each position can be shown in Figure 10 . Under special circumstances, the parameter $Z$ will decrease when the material is blocked in the crushing chamber, as shown in Figure 10(d). The results illustrate that the intervals of the generalized coordinates are contained within the boundaries of the orientation workspace.

According to the algorithms in Section 3.3, the distribution characteristics of the position singularity locus for the 6 -DOF robotic crusher can be drawn in Figure 11. It can be clearly seen that the surface of the singularity in different positions varies greatly, and they are very complex and diverse. Among them, there are three surfaces in each singularity locus, and two holes appear in the center of the most complicated singularity locus, as shown in 


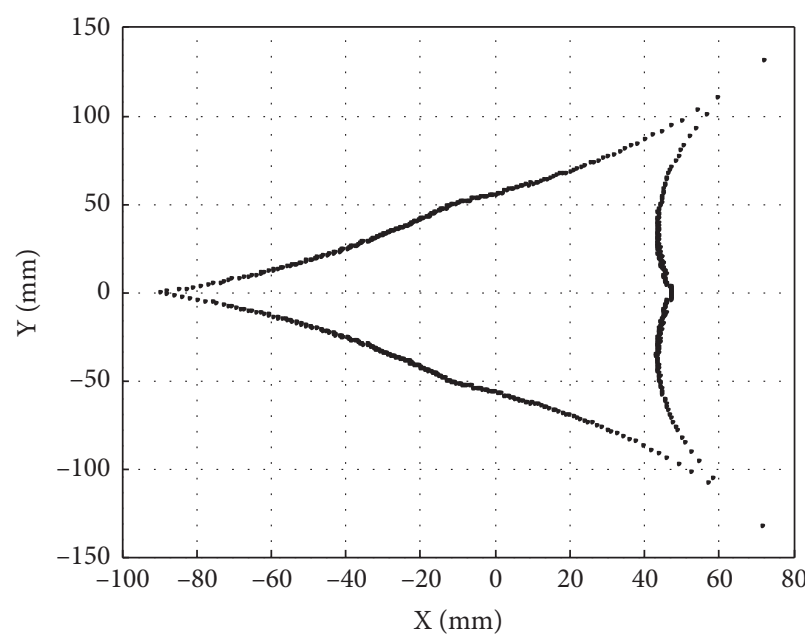

(a)

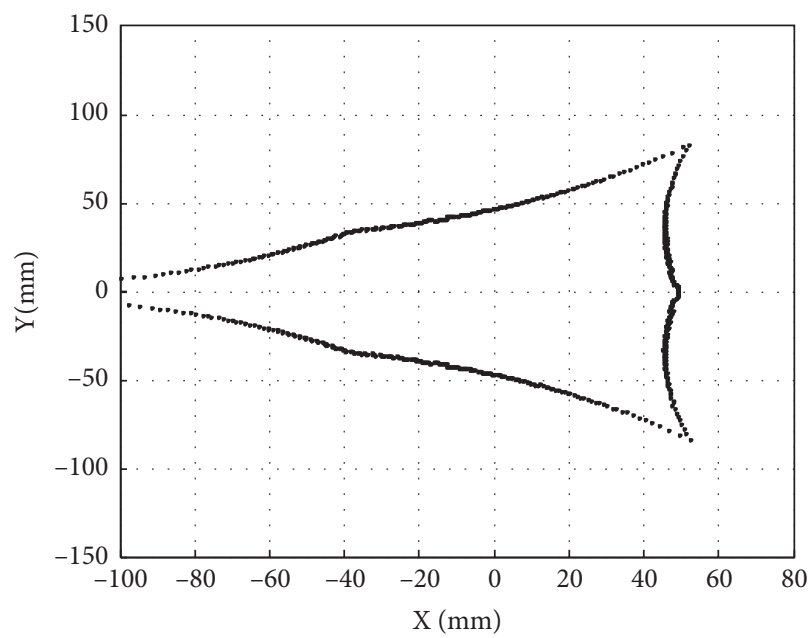

(c)

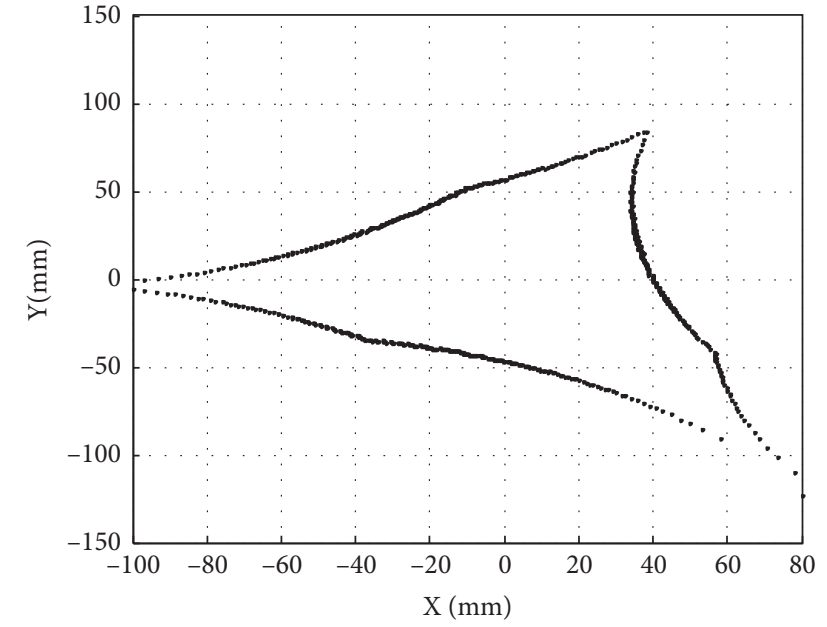

(b)

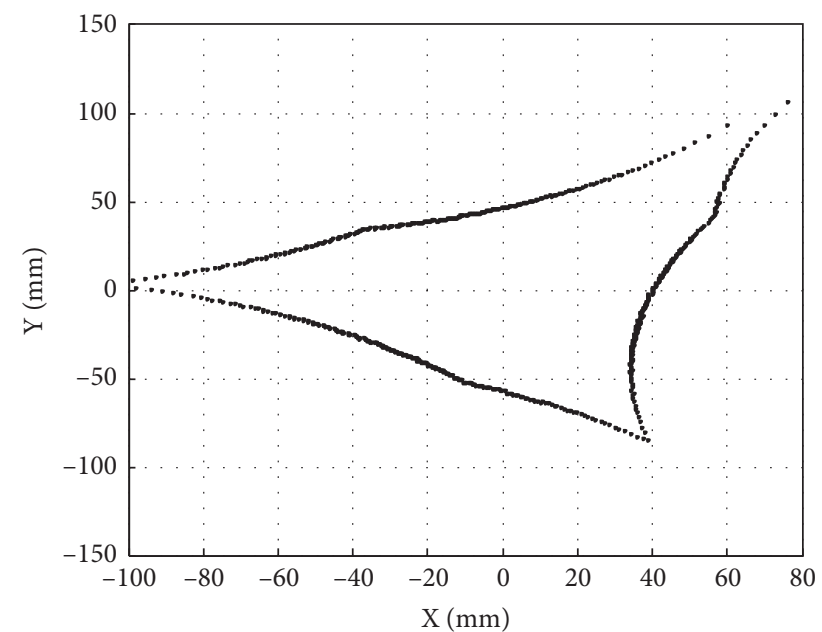

(d)

Figure 9: The boundary curves of position workspace. (a) $\left(\alpha=\gamma=0, \beta=2.52^{\circ},(Z)=-45\right)$. (b) $\left(\alpha=2.52^{\circ}, \beta=0, \gamma=-4.47^{\circ},(Z)=-45\right)$. (c) $\left(\alpha=\gamma=0, \beta=-2.52^{\circ},(Z)=-45\right)$. (d) $\left(\alpha=-2.52^{\circ}, \beta=0, \gamma=4.47^{\circ},(Z)=-45\right)$.

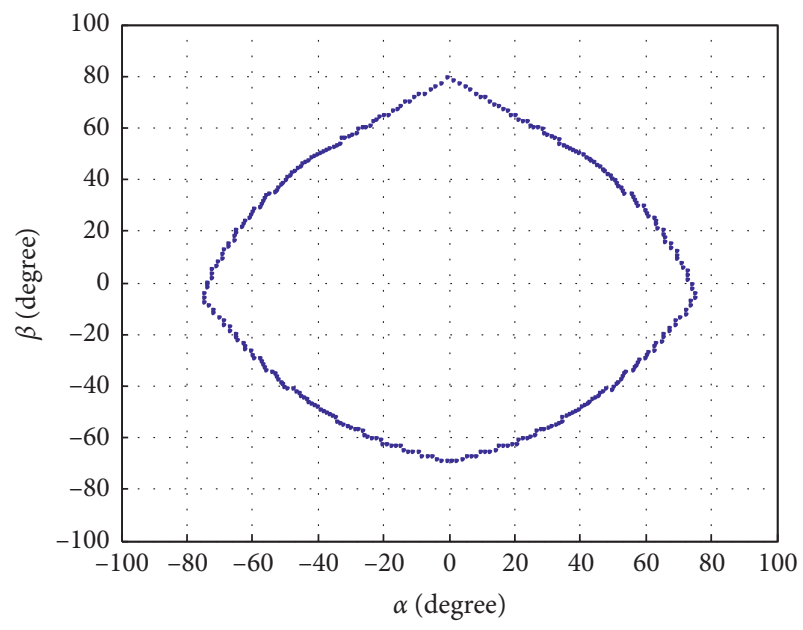

(a)

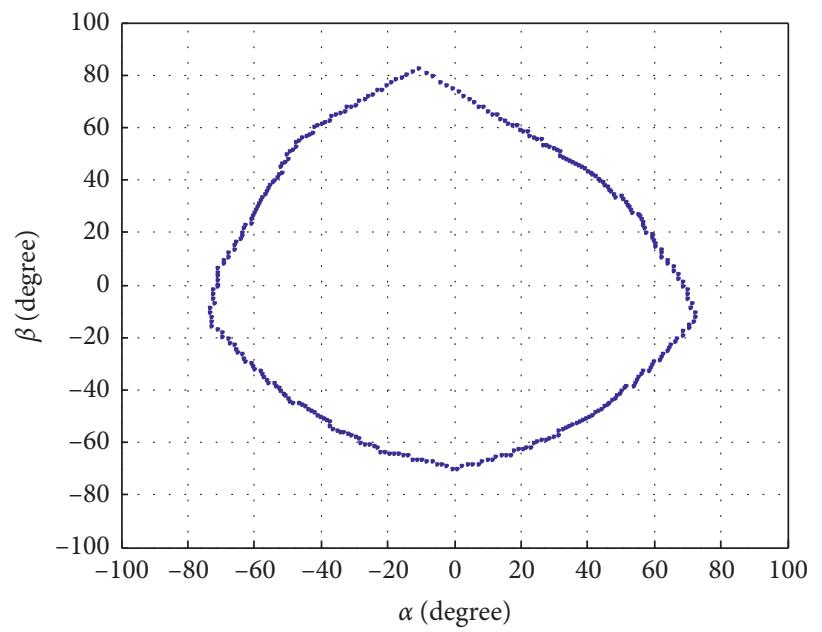

(b)

Figure 10: Continued. 


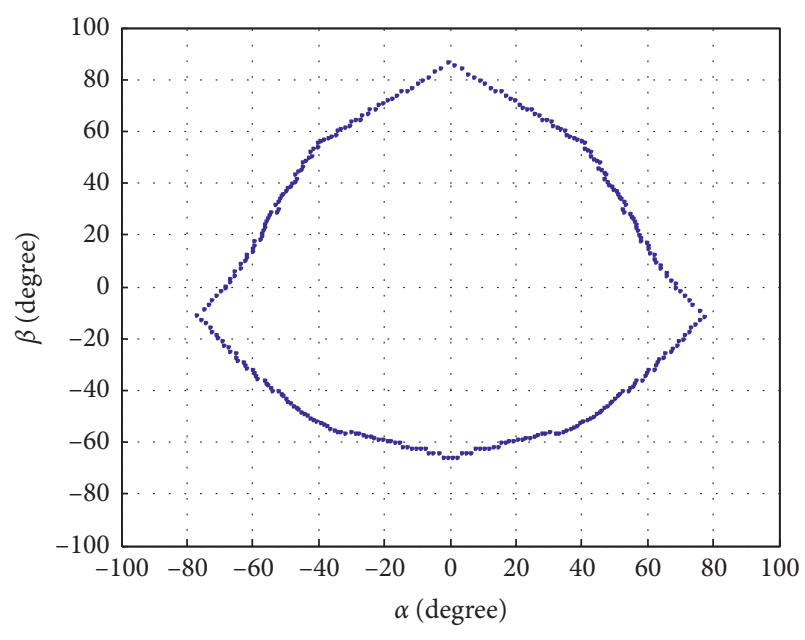

(c)

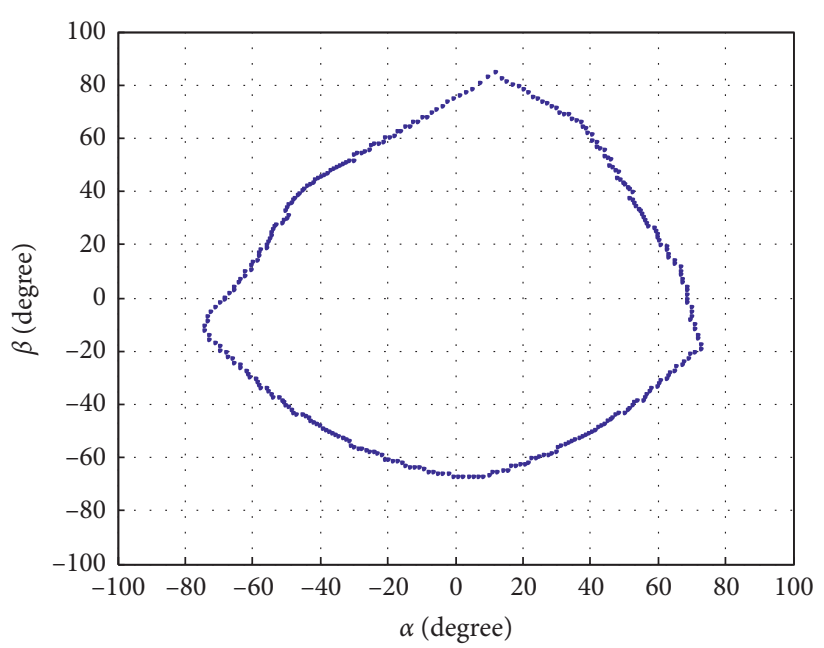

(d)

Figure 10: The boundary curves of orientation workspace. (a) $((X)=9.5,(Y)=(Z)=0, \gamma=0),(\mathrm{b})((X)=-4.9,(Y)=-14.4,(Z)=0$, $\left.\gamma=-4.47^{\circ}\right)$, (c) $((X)=-19.2,(Y)=(Z)=0, \gamma=0),(\mathrm{d})\left((X)=-4.9,(Y)=14.4,(Z)=-10, \gamma=4.47^{\circ}\right)$.

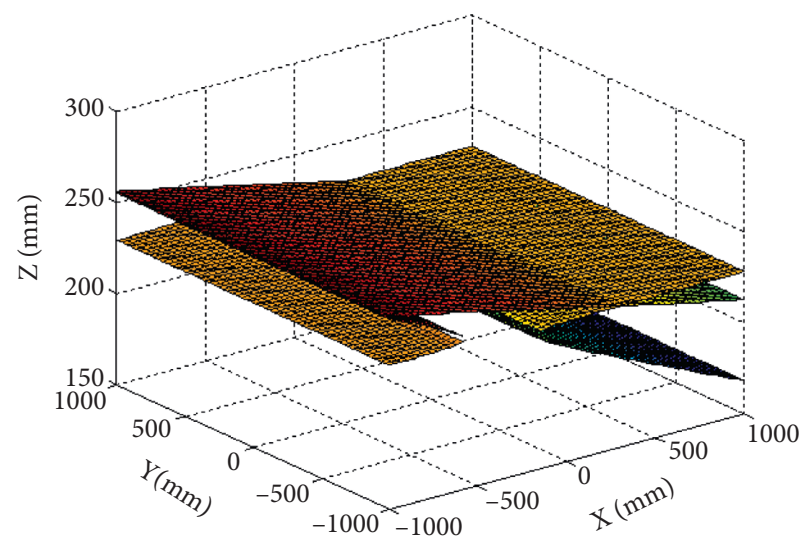

(a)

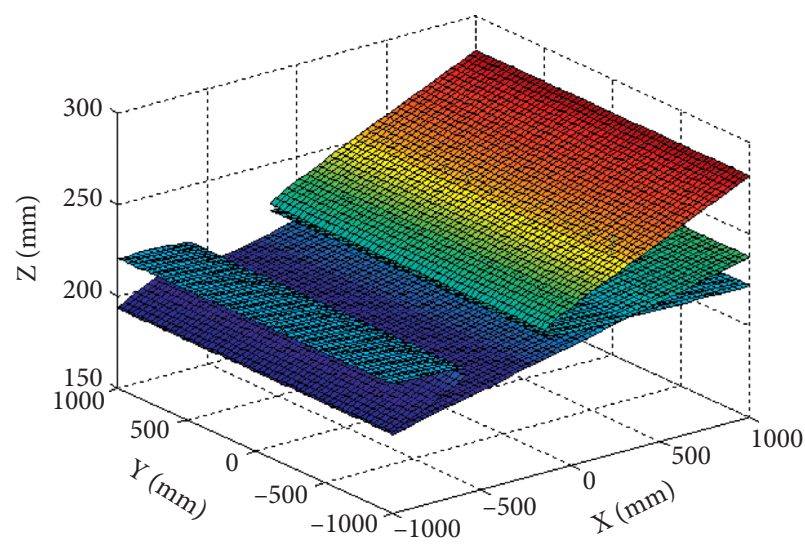

(c)

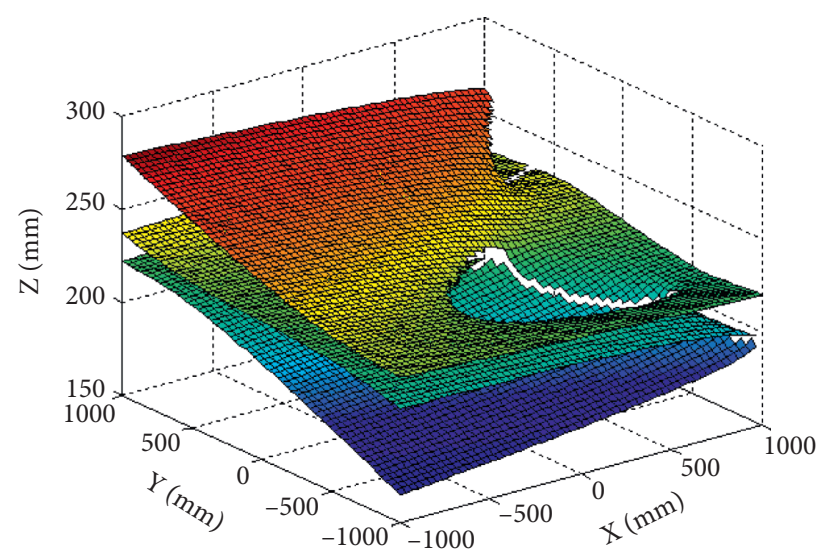

(b)

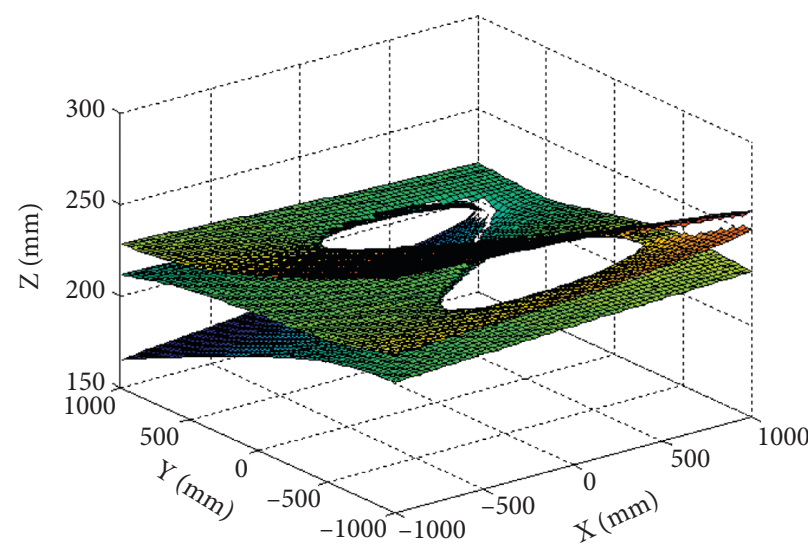

(d)

FIGURE 11: The singularity locus with different orientations. (a) $\left(\alpha=\gamma=0, \beta=2.52^{\circ}\right)$, (b) $\left(\alpha=2.52^{\circ}, \beta=0, \gamma=-4.47^{\circ}\right)$, (c) $(\alpha=\gamma=0$, $\left.\beta=-2.52^{\circ}\right)$, (d) $\left(\alpha=-2.52^{\circ}, \beta=0, \gamma=4.47^{\circ}\right)$. 


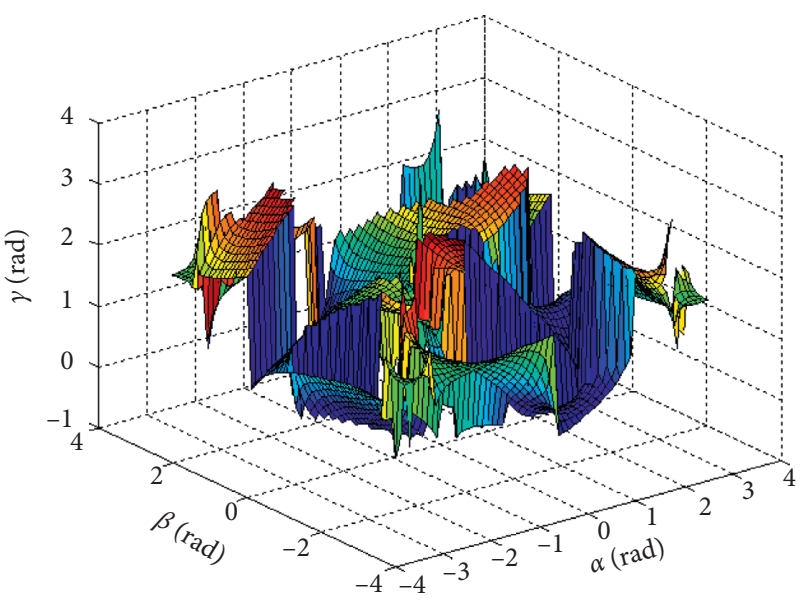

(a)

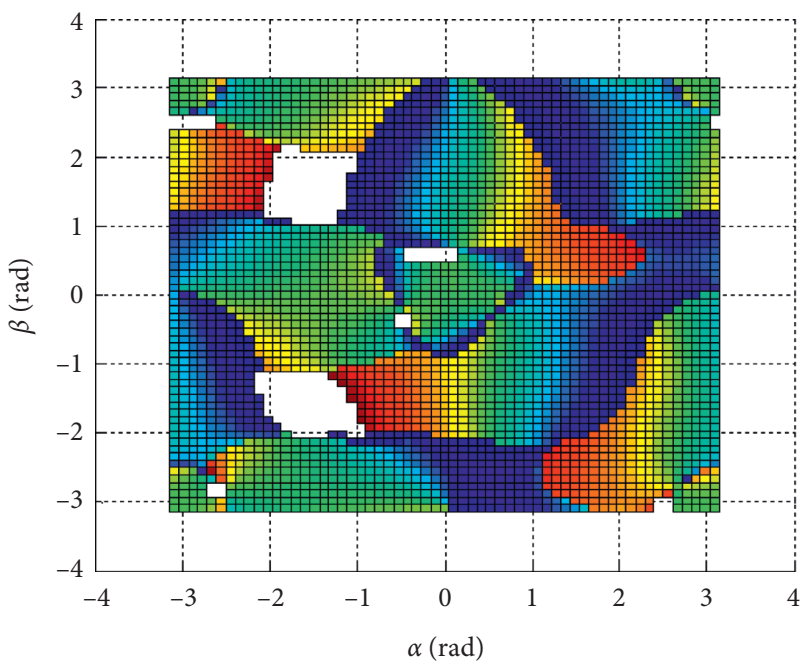

(c)

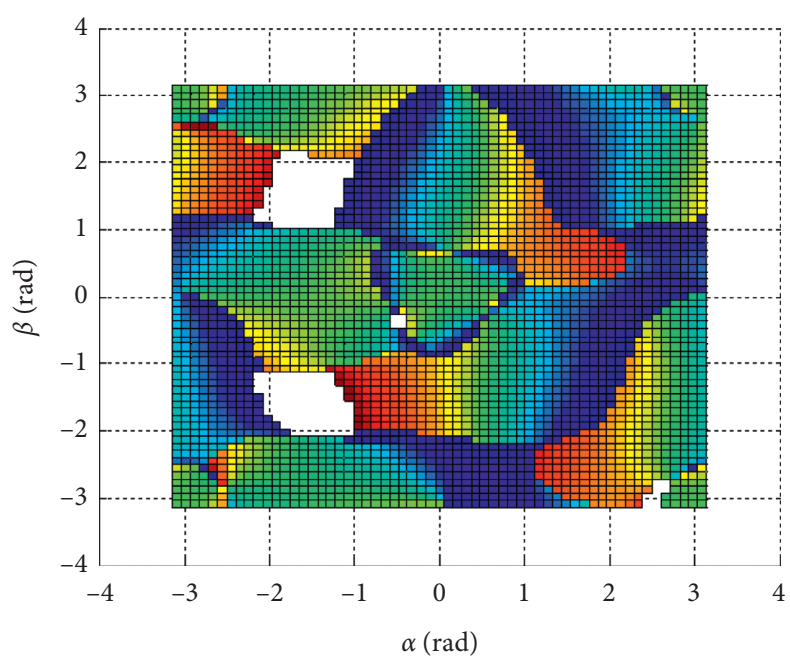

(b)

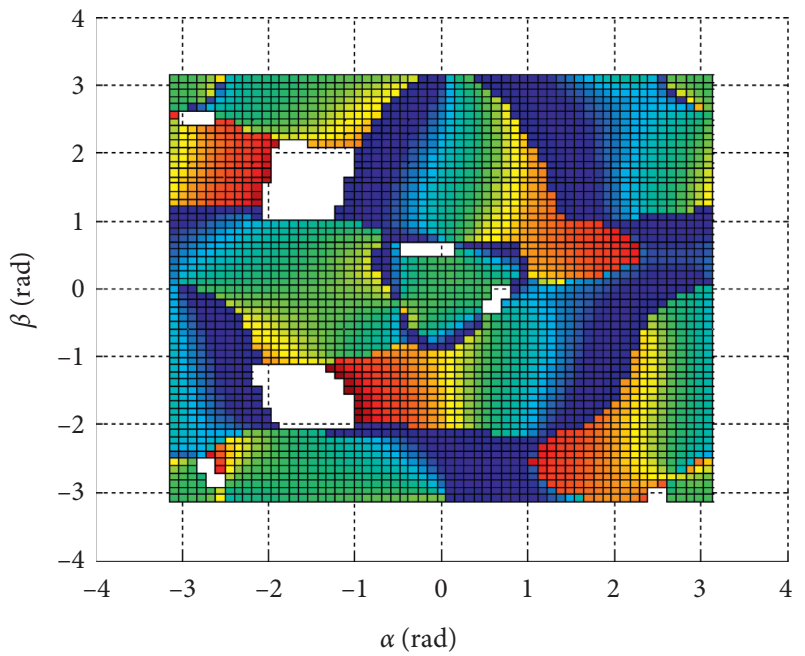

(d)

Figure 12: The singularity locus with different positions. (a) $((X)=9.5,(Y)=(Z)=0)$, (b) $((X)=-4.9,(Y)=-14.4,(Z)=0),(\mathrm{c})((X)=-19.2$, $(Y)=(Z)=0),(d)((X)=-4.9,(Y)=14.4,(Z)=0)$.

Figure 11(d). Considering the trajectory model of the mantle along the $Z$-axis is given as $Z=0$, it is not difficult to find that the position singularity locus has no intersection with the $X Y$ plane passing through the coordinate origin. The results show that the 6-DOF robotic crusher has avoided the position singularity.

Graphical representations of the singularity locus with different positions can be shown in Figure 12. Compared to the position singularity locus, it can be found that the orientation singularity locus is more irregular and complex. Since the trigonometric function has periodicity, the determinant of force Jacobian matrix is very hard to solve. In this case, polynomials instead of trigonometric functions can be used to determine the orientation singularity locus. There exists a nonsingular orientation void around the origin $\left(0^{\circ}, 0^{\circ}, 0^{\circ}\right)$ which is completely inside the orientation singularity locus. The shape of each gap is basically the same and can be described as a triangular region. It can be seen that the intervals of the generalized coordinates are included in the boundaries of the nonsingular orientation void. To sum up, the effectiveness of the optimization design for the 6-DOF robotic crusher is verified by the workspace and singularity of the sampling points.

\section{Conclusions}

In this paper, the structure parameters optimized by a genetic algorithm are proposed based on the transmission index and conditional number. The instantaneous power model of the 6-DOF robotic crusher was established according to the screw theory, and the transmission index was deduced. As a criterion of kinematic accuracy, the condition number related to the Jacobian matrix was derived. Due to the different order of magnitude, two objective functions were transformed into a single-objective function and the corresponding weighting factors were 
applied. In addition, the workspace and singularity locus were determined by geometric constraints and force Jacobian matrix, respectively. Then, based on the trajectory model of the mantle, several sampling positions and orientations were selected to verify the rationality of the optimized structure. It is observed that the intervals of generalized coordinates are included in the boundary curves of the workspace. Meanwhile, the position singularity locus has no intersection with the XY plane passing through the coordinate origin, and there is always a triangular region representing the nonsingular orientation void around the origin $\left(0^{\circ}, 0^{\circ}, 0^{\circ}\right)$. As such, the proposed GA methodology can be considered as a significant contribution to the optimization design for the 6-DOF robotic crusher.

\section{Data Availability}

The data used to support the findings of this study are available from the corresponding author upon request.

\section{Conflicts of Interest}

The authors declare that they have no conflicts of interest.

\section{Acknowledgments}

This work was supported by the National Key Research and Development Program of China (no. 2016YFC0600805).

\section{References}

[1] H. Li, G. R. McDowell, and I. S. Lowndes, "A laboratory investigation and discrete element modeling of rock flow in a chute," Powder Technology, vol. 229, pp. 199-205, 2012.

[2] Z. Zhang, T. Ren, J. Cheng, and X. Jin, "The improved model of inter-particle breakage considering the transformation of particle shape for cone crusher," Minerals Engineering, vol. 112, pp. 11-18, 2017.

[3] Y. Ma, X. Fan, and Q. He, "Prediction of cone crusher performance considering liner wear," Applied Sciences, vol. 6, no. 12, pp. 1-13, 2016.

[4] E. Lee and C. M. Evertsson, "A comparative study between cone crushers and theoretically optimal crushing sequences," Minerals Engineering, vol. 24, no. 3-4, pp. 188-194, 2011.

[5] J. Lichter, K. Lim, A. Potapov, and D. Kaja, "New developments in cone crusher performance optimization," Minerals Engineering, vol. 22, no. 7-8, pp. 613-617, 2009.

[6] X. Huang, Q. Liao, and S. Wei, "Closed-form forward kinematics for a symmetrical 6-6 Stewart platform using algebraic elimination," Mechanism and Machine Theory, vol. 45, no. 2, pp. 327-334, 2010.

[7] I. Miletović, D. M. Pool, O. Stroosma, M. M. Van Paassen, and Q. P. Chu, "Improved Stewart platform state estimation using inertial and actuator position measurements," Control Engineering Practice, vol. 62, pp. 102-115, 2017.

[8] C.-J. Lin and C.-T. Chen, "Reconfiguration for the maximum dynamic wrench capability of a parallel robot," Applied Sciences, vol. 6, no. 3, 2016.

[9] G. Li, B. Shi, and R. Liu, "Dynamic modeling and analysis of a novel 6-DOF robotic crusher based on movement characteristics," Mathematical Problems in Engineering, vol. 2019, Article ID 2847029, 11 pages, 2019.

[10] G. W. Delaney, R. D. Morrison, M. D. Sinnott, S. Cummins, and P. W. Cleary, "DEM modelling of non-spherical particle breakage and flow in an industrial scale cone crusher," Minerals Engineering, vol. 74, pp. 112-122, 2015.

[11] R. Liu, B. Shi, G. Li, and H. Yu, "Influence of operating conditions and crushing chamber on energy consumption of cone crusher," Energies, vol. 11, no. 5, p. 1102, 2018.

[12] W. Li, Y. Yang, W.-d. Shi, X. Zhao, and W. Li, "The correction and evaluation of cavitation model considering the thermodynamic effect," Mathematical Problems in Engineering, vol. 2018, Article ID 7217513, 11 pages, 2018.

[13] J. Wang, C. Wu, and X.-J. Liu, "Performance evaluation of parallel manipulators: motion/force transmissibility and its index," Mechanism and Machine Theory, vol. 45, no. 10, pp. 1462-1476, 2010.

[14] X.-J. Liu, L.-P. Wang, F. Xie, and I. A. Bonev, "Design of a three-axis articulated tool head with parallel kinematics achieving desired motion/force transmission characteristics," Journal of Manufacturing Science and Engineering, vol. 132, no. 2, pp. 1-8, 2010.

[15] X. Chen, "Evaluation of the maximum value of motion/force transmission power in parallel manipulators," Journal of Mechanical Engineering, vol. 50, no. 3, 2014.

[16] Y. Zhao, "Analysis of translational parallel mechanism with good motion/force transmissibility," Journal of Mechanical Engineering, vol. 52, no. 19, 2016.

[17] G. Cui, H. Zhou, N. Wang, and H. Zhang, "Multi-objective optimization of 3-UPS-S parallel mechanism based on isight," Nongye Jixie Xuebao, vol. 44, no. 9, pp. 261-266, 2013.

[18] X. L. Wang, D. J. Zhao, B. Zhang, and W. Li, "Kinematics analysis and optimization design of noval 4-DOF parallel mechanism," Dongbei Daxue Xuebao, vol. 39, no. 4, pp. 532-537, 2018.

[19] M. M. Horoub, M. Hassan, and M. A. Hawwa, "Workspace analysis of a Gough-Stewart type cable marine platform subjected to harmonic water waves," Mechanism and Machine Theory, vol. 120, pp. 314-325, 2018.

[20] H. Li, C. M. Gosselin, M. J. Richard, and B. M. St-Onge, "Analytic form of the six-dimensional singularity locus of the general Gough-Stewart platform," Journal of Mechanical Design, vol. 128, no. 1, pp. 279-287, 2006.

[21] Z. Huang and Y. Cao, "Property identification of the singularity loci of a class of gough-stewart manipulators," The International Journal of Robotics Research, vol. 24, no. 8, pp. 675-685, 2014.

[22] H. Pendar, M. Mahnama, and H. Zohoor, "Singularity analysis of parallel manipulators using constraint plane method," Mechanism and Machine Theory, vol. 46, no. 1, pp. 33-43, 2011.

[23] I. Siti, M. Mjahed, H. Ayad, and A. El Kari, "New trajectory tracking approach for a quadcopter using genetic algorithm and reference model methods," Applied Sciences, vol. 9, no. 9, pp. 1-21, 2019.

[24] C. Zhou, X. Liu, W. Chen, F. Xu, and B. Cao, “Optimal sliding mode control for an active suspension system based on a genetic algorithm," Algorithms, vol. 11, no. 12, 2018.

[25] Y. Al-Douri, H. Hamodi, and J. Lundberg, "Time series forecasting using a two-level multi-objective genetic algorithm: a case study of maintenance cost data for tunnel fans," Algorithms, vol. 11, no. 8, 2018. 
[26] X. K. Han and Z. Zhang, "Topological optimization of phononic crystal thin plate by a genetic algorithm," Scientific Reports, vol. 9, no. 1, p. 8331, 2019.

[27] K. Y. Tsai and J. C. Lin, "Determining the compatible orientation workspace of Stewart-Gough parallel manipulators," Mechanism and Machine Theory, vol. 41, no. 10, pp. 1168$1184,2006$.

[28] I. A. Bonev and J. Ryu, "A geometrical method for computing the constant-orientation workspace of 6-PRRS parallel manipulators," Mechanism and Machine Theory, vol. 36, no. 1, pp. 1-13, 2001.

[29] B. Li, Y. Cao, Q. Zhang, and Z. Huang, "Position-singularity analysis of a special class of the Stewart parallel mechanisms with two dissimilar semi-symmetrical hexagons," Robotica, vol. 31, no. 1, pp. 123-136, 2012.

[30] Y. Cao, Z. Huang, H. Zhou, and W. Ji, “Orientation workspace analysis of a special class of the Stewart-Gough parallel manipulators," Robotica, vol. 28, no. 7, pp. 989-1000, 2010. 